\title{
Bilateral horizontal Vogt's striae in keratoconus
}

\author{
İnci Ulu Güngör, \\ Ümit Beden, \\ Barıș Sönmez \\ Ondokuz Mayıs University, \\ School of Medicine, Department \\ of Ophthalmology, Samsun, Turkey
}

Correspondence: Inci Ulu Güngör

Ondokuz Mayıs Üniversitesi,Tıp Fakültesi,

Göz Hastalıkları A.D., 55139 Kurupelit,

Samsun, Turkey

$\mathrm{Tel}+90362312 \quad 1919 / 2627$

Fax +90362457604 I

Email ligungor@omu.edu.tr
Purpose: To report a keratoconus case with bilateral horizontal Vogt's striae.

Method: The clinical findings of the patient and the development of the direction of striae are discussed.

Results: Vogt's striae, defined as vertical stress lines, are rarely horizontal. One patient with unilateral horizontal stress lines on his left eye has been reported in the literature. Our patient has horizontal Vogt's striae in both eyes.

Conclusion: Horizontal Vogt's striae may be seen in keratoconus as a rare slit-lamp biomicroscopic finding.

Keywords: keratoconus, Vogt's striae, horizontal stress lines

\section{Introduction}

Keratoconus is an ectatic disorder of the cornea. Noninflammatory thinning of the corneal stroma produces a cone-shaped protrusion of the cornea and gradually impairs its optical features. The corneal thinning and conical shape caused by keratoconus induce irregular astigmatism and myopia and impair vision quality (Robinowitz 2005).

In the early stages of keratoconus, the cornea may appear normal. As keratoconus progresses, clinical signs such as Fleischer's ring and Vogt's striae, which are easily demonstrated by slit-lamp biomicroscopy are seen. Vogt's striae are vertical lines in the deep stroma and Descemet's membrane that are parallel to the axis of the cone (Robinowitz 2005). Thirty-five percent of keratoconus patients have Vogt's striae in one eye; 30\% have it in both eyes (Zadnik et al 1998). Only one reported case has horizontal stress lines in one eye (Chung and Kim 2005). In this paper, we report a keratoconus patient who has horizontal Vogt's striae bilaterally and discuss horizontal stress lines.

\section{Material and methods}

Clinical features of a keratoconus patient with bilaterally horizontal Vogt's striae were retrospectively reviewed. His full eye examination was noted. His cornea photograph and cornea topography were taken.

\section{Results}

An 18-year-old male patient was admitted to our clinic with complaints of decreased vision quality and visual acuity for 2 years. He had used spectacles which he needed to replace frequently. He did not have a history of contact lens use. On examination, his best corrected visual acuities were $0.4 \mathrm{OD}$ and $0.1 \mathrm{OS}$ with refractions $-3.00-1.75 \times 70^{\circ}$ and $-6.00-5.75 \times 115^{\circ}$. Slit-lamp examination revealed Fleischer's rings and Vogt's striae in both corneas. Vogt's striae were localized horizontally in the deep stroma and in the Descemet's membrane bilaterally and were more intense on his left cornea (Figure 1). These horizontal lines disappeared when pressure was applied on the globe. Corneal topography (KR.8100P; Topcon, The Netherlands) of both eyes revealed 


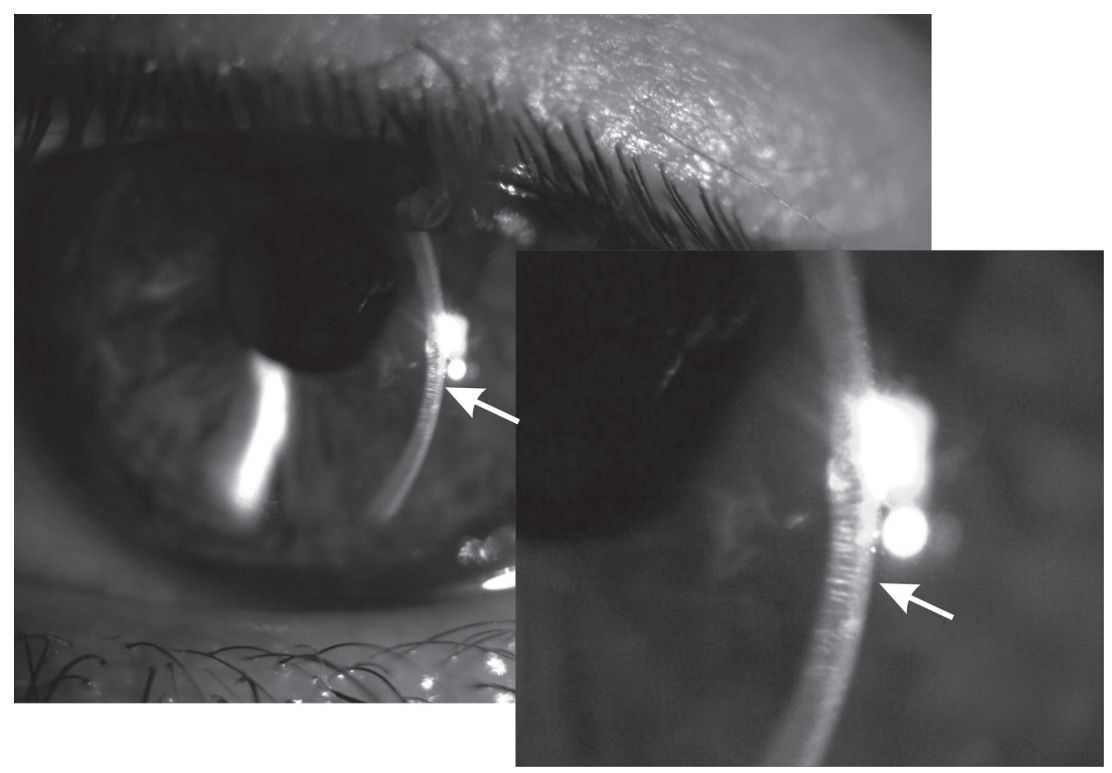

Figure I Horizontal Vogt's striae on the left cornea of the patient.

[OD (Right) Data]

\section{<Ref Data>}

$S:-3.62 \quad \mathrm{C}:-2.62$ A: 56

\section{STerate Datas}

\begin{tabular}{|c|c|c|c|}
\hline & D & MM & A \\
\hline H & 49.62 & 6.80 & 141 \\
\hline v & 46,00 & 7.34 & 51 \\
\hline Ave: & 47,75 & 7,07 & \\
\hline
\end{tabular}

¿Peripheral Datas

$$
\begin{aligned}
& \text { **, }+* \\
& \begin{array}{l|l}
8.08 \quad 8.26
\end{array} \\
& \begin{array}{l}
4.80 \\
-7.92
\end{array} \\
& 7.63 \pm 7.34 \\
& \begin{array}{l|l}
7.94 & 7.18
\end{array} \\
& 6.72
\end{aligned}
$$

\section{[OS (Left) Data]}

\section{Ref Data?}

N.A.

Gerato Data?

\begin{tabular}{rrr} 
D & MH & \multicolumn{1}{c}{$A$} \\
$H=57.25$ & 5.90 & 143 \\
$V=62.12$ & 5.43 & 53 \\
Ave $=59.50$ & 5.67 & \\
CHLt & -4.87 & 143
\end{tabular}

KPeripheral Datas

$$
\begin{gathered}
0.74^{1.60} \\
7.56 .50^{7.54} \\
6.12^{5.43}=6.65
\end{gathered}
$$

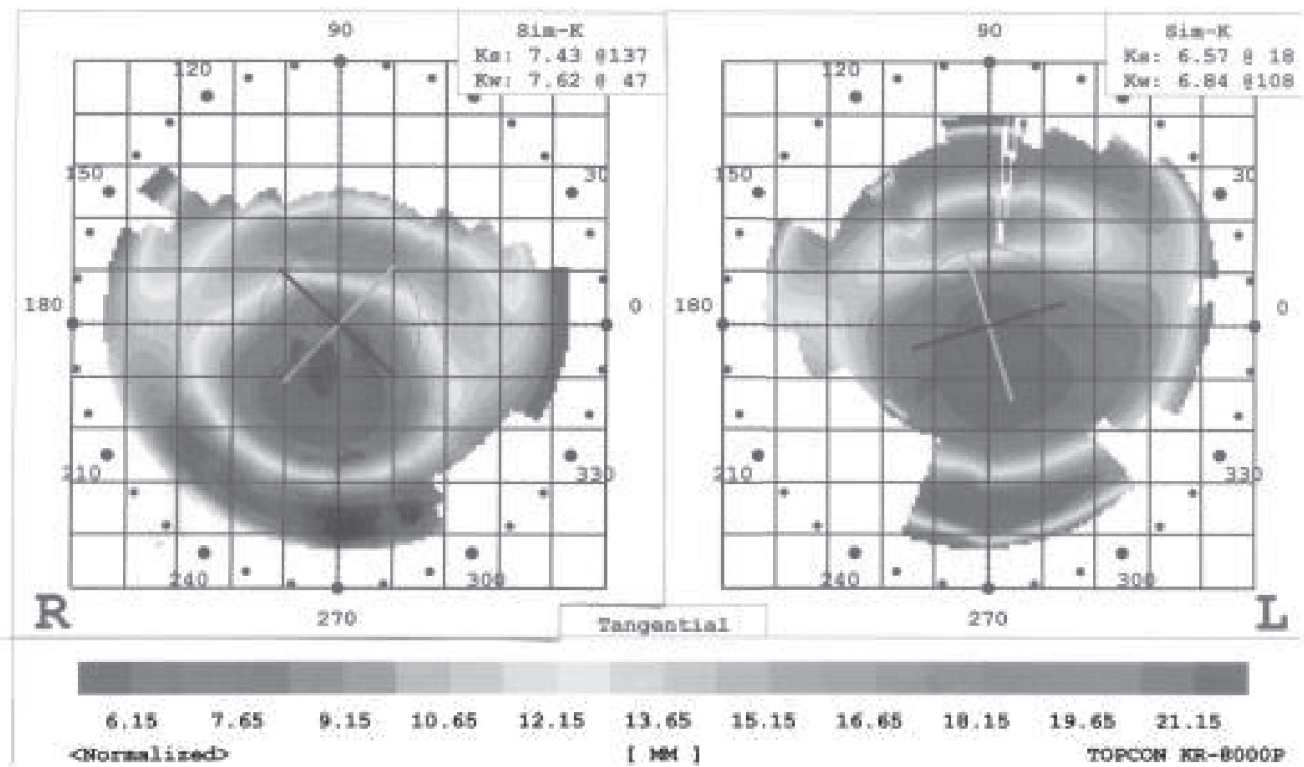

Figure 2 Cone formations in the inferior part of the corneal topography of both eyes. 
steeping of corneal curvatures in the inferior side (Figure 2). The patient was fitted with rigid gas-permeable contact lenses (CFKE; Wöhlk-Zeiss, Germany.); visual acuity increased to 1.0 Snellen lines on OD and 0.8 Snellen lines on OS.

\section{Discussion}

Vogt's striae are common signs of keratoconus and are seen in the deep stroma vertically. They are rarely horizontal. We found only one patient with unilateral Vogt's striae in the literature (Chung and Kim 2005). There are several explanations of the development of Vogt's striae. Hallingsworth and Efron (2005) observed a positive correlation between the orientation of the bands in the posterior stroma and the steepest Sim-K axis of the cornea as determined by corneal topography. They found evidence of bands running horizontally in only one subject of 29 subjects. The authors hypothesized that the direction of bands depend upon a pattern of stress of collagen fibers which are emerged from cone apex. The stress lines run usually vertically because most cones locate inferior to the center of cornea. Uçakhan and colleagues (2006) reviewed the mechanisms of Vogt's striae.
They found endothelial cells toward the cone apex in some eyes with keratoconus. Their long-axis oriented toward the cone apex. Mechanical stretching of endothelial cells and Descemet's membrane may play an important role in this phenomenon. Another explanation may be the distortion of cornea by the cone (Uçakhan et al 2006). These observations suggest that stress lines locate usually vertically according to cone localization. On rare occasions they can also be horizontal as a result of the same mechanisms. Horizontal Vogt's striae may be an occasional sign of keratoconus.

\section{References}

Chung S, Kim EK. 2005. Keratoconus with unilateral horizontal stress lines. Cornea, 24:890.

Hallingsworth JG, Efron N. 2005. Observations of banding patterns (Vogt Striae) in keratoconus. A confocal microscopy study. Cornea, 24:162-6.

Robinowitz YS. 2005. Ectatic disorders of the cornea. In: Foster CS, Azar DT, Dohlman CH (eds). The Cornea. Philadelphia, Lippincott Williams and Wilkins, pp. 889-911.

Uçakhan Ö, Kanpolat A, Yılmaz N, et al. 2006. In vivo confocal microscopy findings in keratoconus. Eye and Contact Lens, 32:183-91.

Zadnik K, Barr JT, Edrington TB, et al. 1998. Baseline findings in the Collaborative Longitudinal Evaluation of Keratoconus (CLEK) Study. Invest Ophthalmol Vis Sci, 39:2537-46. 
